TRANSACTIONS OF THE

AMERICAN MATHEMATICAL SOCIETY

Volume 279. Number 1, September 1983

\title{
ELEMENTARY FIRST INTEGRALS OF DIFFERENTIAL EQUATIONS
}

BY

M. J. PRELLE AND M. F. SINGER

\begin{abstract}
We show that if a system of differential equations has an elementary first integral (i.e. a first integral expressible in terms of exponentials, logarithms and algebraic functions) then it must have a first integral of a very simple form. This unifies and extends results of Mordukhai-Boltovski, Ritt and others and leads to a partial algorithm for finding such integrals.
\end{abstract}

1. Introduction. It is not always possible and sometimes not even advantageous to write the solutions of a system of differential equations explicitly in terms of elementary functions. Sometimes, though, it is possible to find elementary functions that are constant on solution curves, that is, elementary first integrals. These first integrals allow one to occasionally deduce properties that an explicit solution would not necessarily reveal. Consider the following example:

EXAMPLE 1. The preditor-prey equations

$$
\frac{d x}{d t}=a x-b x y, \quad \frac{d y}{d t}=-c y+d x y, \quad a, b, c, d \text { positive real numbers. }
$$

Although these cannot be solved explicitly in finite terms, one can show that

$$
F(x, y)=d x+b y-c \log x-a \log y
$$

is constant on solution curves $(x(t), y(t))$. Using the function $F(x, y)$, one can furthermore show that all solution curves in the positive quadrant are closed, that is, all such solutions are periodic.

Note that in this example the first integral is of the form

$$
w_{0}(x, y)+\sum c_{i} \log w_{i}(x, y) \text {, }
$$

where the $c_{i}$ are constants and the $w_{i}$ are algebraic (in this case, even rational) functions of $x$ and $y$. Roughly speaking, the main result of this paper is that if a system of differential equations has an elementary first integral, it will then have one of this form. Corollaries of the main result will show that the theory presented here unifies and generalizes a number of results originally due to Mordukhai-Boltovski, Ritt and others. An attempt to do this was made in [SING: 77] but the results presented here are more general and the techniques more to the point. Some of these results also appear in [PRELLE: 82]. In the following, $\mathbf{Z}$ stands for the integers, $\mathbf{Q}$ the rationals and $\mathbf{C}$ the complex numbers.

Received by the editors September 8, 1982.

1980 Mathematics Subject Classification. Primary 12H05. 
2. Main result and corollaries. To fix notation, we let $(K, \Delta)$ denote a differential field of characteristic zero with a given set of derivations $\Delta=\{\delta\}_{\delta \in \Delta}$. The constants of $(K, \Delta)$, that is, all those elements annihilated by all $\delta$ in $\Delta$, will be denoted by $C(K, \Delta)$. We assume that the reader is familiar with the definitions of elementary and liouvillian extensions and related notions. For precise definitions see [ROS: 76 or ROSSIN: 77].

The following is our main result. Note that, for $\delta_{1}, \ldots, \delta_{n}$ in $\Delta$, any $K$ linear combination, $y_{1} \delta_{1}+\cdots+y_{n} \delta_{n}, y_{i} \in K$, is a derivation on any $\Delta$-differential extension of $K$.

ThEOREM. Let $(L, \Delta)$ be an elementary extension of the differential field $(K, \Delta)$ with $C(L, \Delta)=C(K, \Delta)$. Let $D=y_{1} \delta_{1}+\cdots+y_{n} \delta_{n}$ for some $\delta_{i} \in \Delta$ and $y_{i} \in K$ and assume that $C(L, \Delta)$ is a proper subset of $C(L,\{D\})$. Then there exist elements of $L$, $w_{0}, w_{1}, \ldots, w_{m}$, algebraic over $K$ and $c_{1}, \ldots, c_{m}$ in $C(K, \Delta)$ such that

$$
D w_{0}+\sum_{i=1}^{m} c_{i} \frac{D w_{i}}{w_{i}}=0 \text { and } \delta w_{0}+\sum_{i=1}^{m} c_{i} \frac{\delta w_{i}}{w_{i}} \neq 0
$$

for some $\delta \in \Delta$.

Let us see how Example 1 fits into this scheme.

EXAMPLE 1 REVISITED. Let $K=\mathbf{C}(x, y)$, $\mathbf{C}$ being the complex numbers, $x$ and $y$ indeterminants. Let $\Delta=\left\{\delta_{x}, \delta_{y}\right\}$ where $\delta_{x}$ (resp. $\delta_{y}$ ) is the partial derivative with respect to $x$ (resp. $y$ ). Let $D=(a x-b x y) \delta_{x}+(-c y+d x y) \delta_{y}$. Let $(L, \Delta)$ be an elementary extension of $(K, \Delta) . L$ then consists of elementary functions of two variables. For $g$ in $L, D g=0$ is equivalent to $g$ being constant on solutions of our system of differential equations. For $g$ in $L, \delta_{x} g \neq 0$ or $\delta_{y} g \neq 0$ is equivalent to $g$ being not identically constant. Therefore, the hypothesis that $C(L, \Delta)$ is properly contained in $C(L,\{D\})$ is equivalent to the existence of a nonconstant elementary function of two variables that is constant on solutions of our equation. The conclusion states that there must exist $w_{0}, w_{1}, \ldots, w_{m}$ algebraic over $\mathbf{C}(x, y)$ such that $w_{0}+\sum_{i=1}^{m} c_{i} \log w_{i}$ is constant on solutions of our system (since

$$
\left.D\left(w_{0}+\sum_{i=1}^{m} c_{i} \log w_{i}\right)=D w_{0}+\sum c_{i} \frac{D w_{i}}{w_{i}}=0\right)
$$

and such that $w_{0}+\sum_{i=1}^{m} c_{i} \log w_{i}$ is not identically constant. Notice that letting $m=2, w_{0}=d x+b y, c_{1}=-c, w_{1}=x, c_{2}=-a$, and $w_{2}=y$ illustrates the conclusion of the Theorem.

This example may lead one to conjecture that the $w_{0}, w_{1}, \ldots, w_{m}$ guaranteed to exist by the Theorem may be chosen to actually lie in $K$, rather than being just algebraic over $K$. This is not necessarily true, even if $K$ is a liouvillian extension of its field of contants.

EXAMPLE 2. Let $k=\mathbf{C}(x)$ with the usual derivation with respect to $x$ which we denote by '. Let $E=k\left\langle\sin ^{-1} x\right\rangle=k\left(\left(1-x^{2}\right)^{1 / 2}, \sin ^{-1} x\right)$ and $K=k\langle y\rangle$ where $y=\left(1-x^{2}\right)^{1 / 2} \sin ^{-1} x$. Notice that $y$ is not algebraic over $k$ and that $y^{\prime}=1-$ $x y /\left(1-x^{2}\right)$ so $K=k(y) . K$ is therefore a purely transcendental extension of $\mathbf{C}$ 
which can be made into a $\Delta$-differential field by letting $\Delta=\left\{\delta_{x}, \delta_{y}\right\}$ where $\delta_{x}$ (resp. $\delta_{y}$ ) is just the usual partial derivative with respect to $x$ (resp. $y$ ). Furthermore, $K$ is a $\Delta$-liouvillian extension of $\mathbf{C}$. Letting $D=\delta_{x}+\left(1-x y /\left(1-x^{2}\right)\right) \delta_{y}$, we see that $D$ and ' agree on $K . E$ is an algebraic (and therefore elementary) extension of $K$. In $E$ we have

$$
D \frac{y}{\sqrt{1-x^{2}}}-i \frac{D\left(x+i \sqrt{1-x^{2}}\right)}{x+i \sqrt{1-x^{2}}}=0
$$

while

$$
\delta_{y} \frac{y}{\sqrt{1-x^{2}}}-i \frac{\delta_{y}\left(x+i \sqrt{1-x^{2}}\right)}{x+i \sqrt{1-x^{2}}}=\frac{1}{\sqrt{1-x^{2}}} \neq 0
$$

Recall that

$$
\int \frac{1}{\sqrt{1-x^{2}}}=\sin ^{-1} x=i \ln \left(x+i \sqrt{1-x^{2}}\right) .
$$

Therefore the conclusion of the Theorem is satisfied. Yet it is not true that there exist $w_{0}, w_{1}, \ldots, w_{m}$ in $K$, such that

$$
D w_{0}+\sum c_{i} \frac{D w_{i}}{w_{i}}=0 \quad \text { and } \quad \delta w_{0}+\sum c_{i} \frac{\delta w_{i}}{w_{i}} \neq 0 \quad \text { for some } \delta \in \Delta .
$$

In fact, it is shown in [ROSSIN: 77, p. 335] that if (1) holds for $w_{0}, w_{1}, \ldots, w_{m}$ in $K$, then $w_{0}, w_{1}, \ldots, w_{m}$ are actually in $\mathrm{C}$ so each $\delta w_{i}=0$ for $\delta \in \Delta$.

Proof of THE TheOREM. We shall prove a seemingly stronger statement: Let $(K, \Delta),(L, \Delta)$ and $D$ be as in the Theorem. Assume that there exist $u_{0}, \ldots, u_{m}$ in $L$ and $d_{1}, \ldots, d_{m}$ in $C(U, \Delta)$ such that

$$
\begin{gathered}
D u_{0}+\sum_{i=1}^{m} d_{i} \frac{D u_{i}}{u_{i}}=0 \quad \text { and } \\
\delta u_{0}+\sum_{i=1}^{m} d_{i} \frac{\delta u_{i}}{u_{i}} \neq 0 \quad \text { for some } \delta \in \Delta .
\end{gathered}
$$

Then there exist $w_{0}, \ldots, w_{n}$ in $L$, algebraic over $K$, and $c_{1}, \ldots, c_{n}$ in $C(K, \Delta)$ such that

$$
D w_{0}+\sum_{i=1}^{m} c_{i} \frac{D w_{i}}{w_{i}}=0
$$

and

$$
\delta w_{0}+\sum_{i=1}^{m} c_{i} \frac{\delta w_{i}}{w_{i}} \neq 0 \text { for some } \delta \in \Delta .
$$

The hypotheses of statement (2) are certainly satisfied if the hypotheses of the Theorem are satisfied. Conversely, if the hypotheses of statement (2) are satisfied then in some elementary extension of $L, w=u_{0}+\sum_{i=1}^{m} c_{i} \log u_{i}$ satisfies $D w=0$ and 
$\delta w \neq 0$ for some $\delta \in \Delta$. Yet it is more convenient to prove statement (2). By induction on the transcendence degree of $L$ over $K$, we may assume that $L$ is an algebraic extension of $K(t)$ where $t$ is transcendental over $K$ and either $\delta t=(\delta v) t$ for some $v$ in $K$ and all $\delta$ in $\Delta$ or $\delta t=\delta v / v$ for some $v \neq 0$ in $K$ and all $\delta$ in $\Delta$.

First assume that there is a $w$ in $L$ such that $D w=0$ and $w$ is not in $K$. We then must have $\delta w \neq 0$ for some $\delta$ in $\Delta$ since $C(K, \Delta)=C(L, \Delta)$. Since $L$ is algebraic over $K(t), w$ is a root of an irreducible polynomial $W^{n}+a_{n-1} W^{n-1}+\cdots+a_{0}$ with the $a_{i}$ in $K(t)$. If $\delta a_{i}=0$ for each $\delta$ in $\Delta$ and each $i, 0 \leqslant i \leqslant n-1$, we would have $\delta w=0$ for each $\delta$ in $\Delta$. Therefore, for some $i, \delta a_{i} \neq 0$. Similarly, if $D a_{i} \neq 0$ for some $i$, we would have $D w \neq 0$, so we have $D a_{i}=0$ for all $i$. Therefore there exists an element $w$ in $K(t)$ such that $D w=0$ and $\delta w \neq 0$ for some $\delta \in \Delta$. If $w$ is in $K$, we would satisfy the conclusion of (2), so we can assume that $w$ is not in $K$. If $\delta t=(\delta v) t$ for some $v$ in $K$ and all $\delta \in \Delta$, we have $D t=(D v) t$. Since $C(K,\{D\}) \varsubsetneqq$ $C(K(t),\{D\})$, Proposition 1.26 of [RISCH: 69] tells us that there exists an integer $n$ and an element $s$ in $K$ such that $D s=n(D v) s$. If $\delta s=n(\delta v) s$ for all $\delta$ in $\Delta$, we would have $\delta\left(s t^{-n}\right)=0$ for all $\delta$ in $\Delta$, which would imply that $t$ is algebraic over $K$, a contradiction. Letting $w_{0}=n v, w_{1}=s$ and $c_{1}=-1$, we have $D w_{1}+c_{1} D w_{1} / w_{1}=0$ and $w_{1}+c_{1} \delta w_{1} / w_{1} \neq 0$ for some $\delta$ in $\Delta$ which gives the conclusion of (2). If $\delta t=\delta v / v$ for some $v \neq 0$ in $K$ and all $\delta$ in $\Delta$, then $D t=D v / v$. Again, since $C(K,\{D\}) \subsetneq C(K(t),\{D\})$, Proposition 1.2a of [RISCH: 69] tells us that there exists an $s$ in $K$ such that $D s=D v / v$. If $\delta s=\delta v / v$ for all $\delta$ in $\Delta$, we would have that $\delta(t-s)=0$ for all $\delta$ in $\Delta$. Since $t$ is not algebraic over $K$, we must have $\delta s \neq \delta v / v$ for some $\delta$ in $\Delta$. Letting $w_{0}=s, w_{1}=v$ and $c_{1}=-1$, we have $D w_{0}+$ $c_{1} D w_{1} / w_{1}=0$ and $\delta w_{0}+c_{1} \delta w_{1} / w_{1} \neq 0$ for some $\delta$ in $\Delta$, which gives the conclusion of (2).

Now assume that if $w$ is in $L$ and $D w=0$ then $w$ is in $K$, that is, $C(K,\{D\})=$ $C(L,\{D\})$. Assume also that the hypotheses of (2) are satisfied. We may furthermore assume that the $d_{i}$ are linearly independent over $\mathbf{Q}$ (otherwise let $e_{1}, \ldots, e_{k}$ be a $\mathbf{Q}$-basis of $\mathbf{Q} d_{1}+\cdots+\mathbf{Q} d_{m}$ such that $d_{i}=(1 / \nu) \sum_{j=1}^{k} \nu_{i j} e_{j}$ with $\nu_{i j}$ and $\nu$ in $\mathbf{Z}$. We then have

$$
\begin{aligned}
& 0=D u_{0}+\sum_{i=1}^{m} d_{i} \frac{D u_{i}}{u_{i}}=D u_{0}+\frac{1}{\nu} \sum_{i=1}^{k} e_{i} \frac{D\left(u_{1}^{\nu_{i 1}} \cdots u_{m}^{\nu_{i m}}\right)}{u_{1}^{\nu_{i 1}} \cdots u_{m}^{\nu_{i m}}}, \\
& 0 \neq \delta u_{0}+\sum_{i=1}^{m} d_{i} \frac{\delta u_{i}}{u_{i}}=\delta u_{0}+\frac{1}{\nu} \sum_{i=1}^{k} e_{i} \frac{\delta\left(u_{1}^{\nu_{i 1}} \cdots u_{m}^{\nu_{i m}}\right)}{u_{1}^{\nu_{i 1}} \cdots u_{m}^{\nu_{i m}}}
\end{aligned}
$$

and we may use these equations instead of those in the hypotheses of (2)). If we have $\delta t=(\delta v) t$ for all $\delta$ in $\Delta$, we then have that $D t=(D v) t$. Applying Theorem 2 of [ROS: 76], we have that $u_{0}$ is algebraic over $K$ and that there exist integers $\nu_{0}, \nu_{1}, \ldots, \nu_{n}$ with $\nu_{0} \neq 0$ such that each $u_{i}^{\nu_{i}} / t^{\nu_{0}}$ is algebraic over $K$. Let

$$
w_{0}=u_{0}+\frac{1}{\nu_{0}} \sum_{i=1}^{m} d_{i} \nu_{i} w_{i}, \quad w_{i}=\frac{u_{i}^{\nu_{0}}}{t^{\nu_{0}}} \quad \text { for } i=1, \ldots, m, \quad c_{i}=\frac{1}{\nu_{0}} d_{i} .
$$

We then have

$$
D w_{0}+\sum_{i=1}^{m} c_{i} \frac{D w_{i}}{w_{i}}=D u_{0}+\sum_{i=1}^{m} d_{i} \frac{D u_{i}}{u_{i}}=0
$$


while

$$
\delta w_{0}+\sum_{i=1}^{m} c_{i} \frac{\delta w_{i}}{w_{i}}=\delta u_{0}+\sum_{i=1}^{m} d_{i} \frac{\delta u_{i}}{u_{i}} \neq 0 \text { for some } \delta \text { in } \Delta .
$$

This gives the conclusion of (2). If $\delta t=\delta v / v$ for all $\delta$ in $\Delta$, then $D t=D v / v$. Applying Theorem 2 of [ROS: 76] again we have that $u_{1}, \ldots, u_{m}$ are algebraic over $K$, and that there exists a $c$ in $K$ such that $D c=0$ and such that $u_{0}-c t$ is algebraic over $K$. If $\delta c \neq 0$ for some $\delta$ in $\Delta$ we would be done so we can assume $c$ is in $C(K, \Delta)$. Let

$$
\begin{gathered}
w_{0}=u_{0}-c t, \quad w_{i}=u_{i} \text { for } i=1, \ldots, m, \\
w_{m+1}=v, \quad c_{i}=d_{i} \text { for } i=1, \ldots, m, \\
c_{m+1}=c .
\end{gathered}
$$

We then have

$$
D w_{0}+\sum_{i=1}^{m+1} c_{i} \frac{D w_{i}}{w_{i}}=D u_{0}+\sum_{i=1}^{m} c_{i} \frac{D u_{i}}{u_{i}}=0
$$

while

$$
\delta w_{0}+\sum_{i=1}^{m+1} c_{i} \frac{\delta w_{i}}{w_{i}}=\delta u_{0}+\sum_{i=1}^{m} d_{i} \frac{\delta u_{i}}{u_{i}} \neq 0 \text { for some } \delta \text { in } \Delta .
$$

This gives the conclusion of (2) and finishes the proof.

We will now deduce some corollaries. Corollary 1 is a generalization of a theorem of Mordukhai-Boltovski [M-B: 06] (also see [RITT: 48]), which states: Let $y^{\prime}=$ $f(x, y)$ be a differential equation with $f$ an algebraic function of $x$ and $y$. If there exists an elementary function $g(x, y)$ which is constant on solutions of $y^{\prime}=f(x, y)$, then there exist algebraic functions of two variables $\phi_{0}, \ldots, \phi_{m}$ and constants $c_{1}, \ldots, c_{m}$ such that $\phi_{0}(x, y)+\sum_{i=1}^{m} c_{i} \phi_{i}(x, y)$ is a first integral of $y^{\prime}=f(x, y)$, that is, it is not identically constant but is constant on all solutions of $y^{\prime}=f(x, y)$. By a differential field of functions in $n+1$ variables $x_{0}, x_{1}, x_{2}, \ldots, x_{n}$, we mean a field of functions, meromorphic in some domain in $\mathbf{C}^{n+1}$, closed under the derivations $\partial / \partial x_{i}$ and containing the coordinate functions $x_{1}, \ldots, x_{n}$.

COROLlaRY 1. Let $K$ be a differential field of functions in $n+1$ variables and $L$ an elementary extension of $K$. Let $f$ be in $K$ and assume there exists a nonconstant $g$ in $L$ such that $g$ is constant on all solutions of $y^{(n)}=f\left(x, y, y^{\prime}, \ldots, y^{(n-1)}\right)$. Then there exist $w_{0}, \ldots, w_{m}$ algebraic over $K$ and constants $c_{1}, \ldots, c_{n}$ such that

$$
w_{0}\left(x, y, y^{\prime}, \ldots, y^{(n-1)}\right)+\sum_{i=1}^{m} c_{i} \log w_{i}\left(x, y, y^{\prime}, \ldots, y^{(n-1)}\right)
$$

is constant on all solutions of $y^{(n)}=f\left(x, y, y^{\prime}, \ldots, y^{(n-1)}\right)$.

Proof. Let $D=\partial / \partial x_{0}+x_{2} \partial / \partial x_{1}+x_{3} \partial / \partial x_{2}+\cdots+f \partial / \partial x_{n}$ and apply the Theorem, noting that $D g=0$ if and only if $g$ is constant on all solutions of $y^{(n)}=f\left(x, y, y^{\prime}, \ldots, y^{(n-1)}\right)$.

Loosely speaking, the next result says that if $u_{1}, \ldots, u_{n}$ are elementary functions of a single variable $x$ and $g\left(x, U_{1}, \ldots, U_{n}\right)$ is an elementary function of $n+1$ variables 
$x, U_{1}, \ldots, U_{n}$, such that $g\left(x, \int u_{1} d x, \ldots, \int u_{n} d x\right)$ is constant, then some nontrivial linear combination with constant coefficients of the $\int u_{i}$ is elementary. For $n=1$, this result is due to [RITT: 23, RITT: 48]. The result also appears in [SING: 77] and includes the result of [MOZI: 79]. To be precise, we let $k$ be an ordinary differential field with derivation $\delta$ and let $u_{1}, \ldots, u_{n}$ be elements of $k$. Define a new differential field $(K, \Delta)$ as follows: let $K=k\left(U_{1}, \ldots, U_{n}\right)$ where $U_{1}, \ldots, U_{n}$ are indeterminants. Let $\Delta=\left\{\delta_{0}, \ldots, \delta_{n}\right\}$ where:

(1) $\delta_{0}$ restricted to $k$ is $\delta$ and $\delta_{0} U_{i}=0$ for $i=1, \ldots, n$.

(2) For $i=1, \ldots, n$, let $\delta_{i} a=0$ for all $a$ in $k$ and let $\delta_{i} U_{i}=0$ if $i \neq j$ and $\delta_{i} U_{i}=1$.

COROLlaRY 2. Let $(K, \Delta)$ be as above and let $(L, \Delta)$ be an elementary extension of $(K, \Delta)$ so that $C(L, \Delta)=C(K, \Delta)$. Let $D=\delta_{0}+\sum_{i=1}^{n} u_{i} \delta_{i}$ and assume $C(L, \Delta)$ is properly contained in $C(L,\{D\})$. Then there exist $v_{0}, \ldots, v_{m}$ in $k$, and constants $c_{1}, \ldots, c_{n}, d_{1}, \ldots, d_{m}$ in $C(k, \Delta)$, not all the $c_{i}$ 's being zero, so that

$$
\sum_{i=1}^{n} c_{i} u_{i}=\delta v_{0}+\sum_{i=1}^{m} d_{i} \frac{\delta v_{i}}{v_{i}}
$$

To prove this corollary we need the following lemma. We assume that the reader is familiar with the notation of Theorem 1 of [ROS: 76].

Lemma. Let $(K, \Delta)$ and $D$ be as above and let $(E, \Delta)$ be an algebraic extension of $K$ with $C(E, \Delta)=C(K, \Delta)$. If there exists an $\alpha \in E$, such that $\alpha \notin k$ and $D \alpha=0$ then there exist $c_{1}, \ldots, c_{n} \in C(k,\{\delta\})$ and $w \in k$ such that $\sum_{i=1}^{n} c_{i} u_{i}=\delta w$.

Proof. Let $\alpha \in E$ such that $\alpha \notin k$ and $D \alpha=0$. Let $x^{m}+a_{m-1} x^{m-1}+\cdots+a_{0}$ be the minimum polynomial of $\alpha$ over $k\left(U_{1}, \ldots, U_{n}\right)$. Since $D \alpha=0$, we have $D a_{i}=0$ for $i=0, \ldots, m-1$. If each $a_{i} \in k$, then $\alpha$ would be algebraic over $k$ and so $0=D \alpha=\delta \alpha$ and $\delta_{i} \alpha=0$ for $i=1, \ldots, n$. Since $C(E, \Delta)=C(K, \Delta) \subset k, \alpha$ would be in $k$, a contradiction. We can conclude that for some $a_{i}, a_{i} \notin k$ and $D a_{i}=0$, and therefore we can assume that $\alpha \in k\left(U_{1}, \ldots, U_{n}\right)$. Proceeding by induction on $n$, we can assume that $C(k,\{D\})=C\left(k\left(U_{1}, \ldots, U_{n-1}\right)\{D\}\right)$ while $C(k,\{D\}) \subsetneq$ $C\left(k\left(U_{1}, \ldots, U_{n}\right),\{D\}\right)$. Since $D U_{n}=u_{n}$ which is in $k\left(U_{1}, \ldots, U_{n-1}\right)$, we can conclude that there is an element $V$ in $k\left(U_{1}, \ldots, U_{n-1}\right)$ such that $D V=u_{n}$. Applying Theorem 1 of [ROS: 76$]$ to

$$
\begin{gathered}
D U_{1} \in k \\
\vdots \\
D U_{n-1} \in k \\
D V \in k
\end{gathered}
$$

while noting that $\operatorname{tr} \operatorname{deg} k\left(U_{1}, \ldots, U_{n-1}, V\right) / k=n-1$ we can conclude that the $n$ elements $d U_{1}, \ldots, d U_{n-1}, d V$ of $\Omega_{k\left(U_{1}, \ldots, U_{n-1}, V\right) / k}$ are linearly dependent over $C(k,\{D\})=C(k,\{\delta\})$. Therefore, for some $c_{1}, \ldots, c_{n}$ in $C(k,\{\delta\})$ we have $0=$ $c_{1} d U_{1}+\cdots+c_{n-1} d U_{n-1}+c_{n} d V=d\left(c_{1} U_{1}+\cdots+c_{n} V\right)$. Therefore, $w=c_{1} U_{1}$ $+\cdots+c_{n} V$ is algebraic over $k$ and so in $k$. We also have

$$
D\left(c_{1} U_{1}+\cdots+c_{n} V\right)=c_{1} u_{1}+\cdots+c_{n-1} u_{n-1}+c_{n} u_{n}=D w=\delta w .
$$


Proof of Corollary 2. We can conclude from the Theorem that there are $w_{0}, \ldots, w_{n}$ in $L$, algebraic over $K$, such that

$$
\begin{gathered}
D w_{0}+\sum_{i=1}^{m} c_{i} \frac{D w_{i}}{w_{i}}=0 \\
\delta w_{0}+\sum_{i=1}^{m} c_{i} \frac{\delta w_{i}}{w_{i}} \neq 0 \quad \text { for some } \delta \in \Delta .
\end{gathered}
$$

As in the proof of the Theorem we can assume that the $c_{i}$ are linearly independent over $\mathbf{Q}$.

Letting $E=K\left(w_{0}, \ldots, w_{n}\right)$, we see that $E$ is a differential extension of $K$. Furthermore, we can assume that $C(E,\{D\})=C(k,\{D\})$, since otherwise the Lemma would imply we were done. We now apply Theorem 1 of [ROS: 76] to the $n+1$ equations

$$
\begin{array}{r}
D U_{1} \in k \\
\vdots \\
D U_{n} \in k \\
\sum_{i=1}^{m} c_{i} \frac{D w_{i}}{w_{i}}+D w_{0} \in k
\end{array}
$$

and noting that $\operatorname{tr} \operatorname{deg} k\left(U_{1}, \ldots, U_{n}, w_{0}, \ldots, w_{n}\right) / k<n+1$, conclude that there exist constants $f_{i} \in C(k,\{D\})$ such that

$$
f_{1} d U_{1}+\cdots+f_{n} d U_{n}+f_{n+1}\left(\sum_{i=1}^{m} c_{i} \frac{d w_{i}}{w_{i}}+d w_{0}\right)=0 .
$$

If $f_{n+1}=0$, we have $d\left(f_{1} U_{1}+\cdots+f_{n} U_{n}\right)=0$, so $v_{0}=f_{1} U_{1}+\cdots+f_{n} U_{n}$ is algebraic over $k$ (and therefore in $k$, since $k$ is relatively algebraically closed in $K$ ). Therefore

$$
f_{1} u_{1}+\cdots+f_{n} u_{n}=D\left(f_{1} U_{1}+\cdots+f_{n} U_{n}\right)=D v_{0}=\delta v_{0}
$$

which gives us the conclusion of the corollary.

If $f_{n+1} \neq 0$, then we can conclude that $f_{1} U_{1}+\cdots+f_{n} U_{n}+f_{n+1} w_{0}$ and all the $w_{i}$, $i=1, \ldots, m$, are algebraic over $k$. For some $i, 1 \leqslant i \leqslant n$, we must have $f_{i} \neq 0$. If not, we would have $w_{0}, w_{1}, \ldots, w_{n}$ algebraic over $k$. Since $D$ restricted to $k$ is $\delta$ and each $\delta_{j}, 1 \leqslant i \leqslant n$, restricted to $k$ is 0 , we have $D w_{i}=\delta w_{i}$ for $i=0, \ldots, m$ and $\delta_{j} w_{i}=0$ for $1 \leqslant j \leqslant n, \quad 0 \leqslant i \leqslant m$. This would contradict the relations in (3). Let $k_{1}=$ $k\left(f_{1} U_{1}+\cdots+f_{n} U_{n}+f_{n+1} w_{0}, w_{1}, \ldots, w_{m}\right)$ and let

$$
v_{0}=\operatorname{Trace}\left(f_{1} U_{1}+\cdots+f_{n} U_{n}+f_{n+1} w_{0}\right) \quad \text { and } \quad v_{i}=\operatorname{Norm}\left(w_{i}\right) \text { for } i=1, \ldots, m \text {, }
$$

where the Trace and Norm are taken in the field $k_{1}$ with respect to $k$. We then have, for some integer $p$,

$$
\begin{aligned}
& \frac{D v_{i}}{v_{i}}=p \frac{D w_{i}}{w_{i}}, \quad i=1, \ldots, m, \\
& D v_{0}=p D\left(f_{1} U_{1}+\cdots+f_{n} U_{n}+f_{n+1} w_{0}\right)=p f_{1} u_{1}+\cdots+p f_{n} u_{n}+p f_{n+1} D w_{0} .
\end{aligned}
$$


Since $D w_{0}=-\Sigma c_{i} D w_{i} / w_{i}$ we have

$$
\begin{aligned}
p f_{1} u_{1}+\cdots+p f_{n} u_{n} & =D v_{0}-p f_{n+1} D w_{0} \\
& =D v_{0}+p f_{n+1}\left(\sum_{i=1}^{m} c_{i} \frac{D w_{i}}{w_{i}}\right)=D v_{0}+f_{n+1} \sum_{i=1}^{m} c_{i} \frac{D v_{i}}{v_{i}} .
\end{aligned}
$$

This gives us the conclusion of Corollary 2 .

Finally, note that the results of [MACK: 76] show how one can decide if there exist constants $c_{1}, \ldots, c_{n}$, not all zero such that $\sum c_{i} u_{i}$ has an elementary antiderivative, where the $u_{i}$ lie in a purely transcendental elementary extension of $C(x)$.

In the next corollary, which generalizes a result in [SING: 75], we will focus on the differential equation $y^{\prime}=f(y)$ where $f(y)$ is a nonzero function of one variable. Loosely speaking this corollary says that if $y^{\prime}=f(y)$ has an elementary first integral then

$$
g(y)=\int \frac{1}{f(y)} d y
$$

is an elementary function of $y$. The converse is also true, since if $g(y)$ is an elementary function of $y$ and $y(x)$ is a solution of $y^{\prime}=f(y)$ then $(g(y(x)))^{\prime}=1$ so $g(y)-x$ is an elementary function of $x$ and $y$ which is constant on solutions of $y^{\prime}=f(y)$. If $f(y)$ is an elementary function of $y$, the Risch integration algorithm allows us to decide if $g(y)$ is elementary and so allows us to determine if the differential equation $y^{\prime}=f(y)$ has an elementary first integral.

Suppose we have a differential equation $y^{\prime}=f(y)$, where $f \neq 0$. We model this situation in the following way: let $\left(K,\left\{\delta_{y}\right\}\right)$ be a differential field such that $K$ is a field of functions in the single variable $y$ which contains the element $f(y)$ and $\delta_{y}$ is a derivation of $K$ such that $\delta_{y}(y)=1$. We can extend $K$ to the field $K(x)$ where $x$ is transcendental over $K$. We extend $\delta_{y}$ to a derivation on this field by letting $\delta_{y}(x)=0$ and define a new derivation $\delta_{x}$ on $K(x)$ by letting $\delta_{x}(a)=0$ for all $a$ in $K$ and $\delta_{x}(x)=1$. Let $\Delta=\left\{\delta_{x}, \delta_{y}\right\}$ and $D=\delta_{x}+f \delta_{y}$.

Corollary 3. Let $(K(x), \Delta)$ and $D$ be as above and let $(L, \Delta)$ be an elementary extension of $(K(x), \Delta)$ such that $C(K(x), \Delta)=C(L, \Delta)$. Furthermore, assume $C(K(x),\{D\})$ is a proper subset of $C(L,\{D\})$. Then there exist $u_{0}, u_{1}, \ldots, u_{m}$ in $K$, with $u_{1}, \ldots, u_{m}$ nonzero and $a_{1}, \ldots, a_{n}$ in $C\left(K,\left\{\delta_{y}\right\}\right)$ such that

$$
\frac{1}{f}=\delta_{y} u_{0}+\sum_{i=1}^{m} a_{i} \frac{\delta_{y} u_{i}}{u_{i}} .
$$

Proof. By the Theorem we know that there exist $w_{0}, w_{1}, \ldots, w_{m}$ in $L$, algebraic over $K(x)$ and $c_{1}, \ldots, c_{m}$ in $C(K(x), \Delta)$ such that

$$
D w_{0}+\sum_{i=1}^{m} c_{i} \frac{D w_{i}}{w_{i}}=0
$$

while for some $\delta$ in $\Delta$

$$
\delta w_{0}+\sum_{i=1}^{m} c_{i} \frac{\delta w_{i}}{w_{i}} \neq 0
$$


By an argument given previously, we may assume the $c_{i}$ are linearly independent over $\mathbf{Q}$. Since $\delta x \in K$ for $\delta \in \Delta$, we may apply Theorem 2 of [ROS: 76]. We can conclui 2 that $w_{1}, \ldots, w_{m}$ are algebraic over $K$ and that there exists a $c$ in $C(K, \Delta)=$ $C\left(K,\left\{\delta_{y}\right\}\right)$ such that $w_{0}-c x$ is algebraic over $K$. Let $v_{0}=w_{0}-c x$ and $v_{i}=w_{i}$. We then have

$$
c D x+D v_{0}+\sum_{i=1}^{m} c_{i} \frac{D v_{i}}{v_{i}}=0
$$

while for some $\delta$ in $\Delta$

$$
c \delta x+\delta v_{0}+\sum_{i=1}^{m} c_{i} \frac{\delta v_{i}}{v_{i}} \neq 0 .
$$

Now we shall show that $c \neq 0$. Assume not, then

$$
\begin{aligned}
0= & D v_{0}+\sum_{i=1}^{m} c_{i} \frac{D v_{i}}{v_{i}}=\delta_{x} v_{0} \\
& +\sum_{i=1}^{m} c_{i} \frac{\delta x v_{i}}{v_{i}}+f\left(\delta_{y} v_{0}+\sum_{i=1}^{m} c_{i} \frac{\delta_{y} v_{i}}{v_{i}}\right)=f\left(\delta_{y} v_{0}+\sum_{i=1}^{m} c_{i} \frac{\delta_{y} v_{i}}{v_{i}}\right)
\end{aligned}
$$

since $\delta_{x} v_{0}=\delta_{x} v_{1}=\cdots=\delta_{x} v_{m}=0$. We therefore have

$$
c \delta x+\delta v_{0}+\sum_{i=1} c_{i} \frac{\delta v_{i}}{v_{i}}=\delta v_{0}+\sum_{i=1}^{m} \frac{\delta v_{i}}{v_{i}}=0
$$

for each $\delta$ in $\Delta$, contradicting (5).

Using the fact that $D=\delta_{x}+f \delta_{y}$ we can rewrite (4) as

$$
0=c D x+D v_{0}+\sum_{i=1}^{m} c_{i} \frac{D v_{i}}{v_{i}}=c+f\left(\delta_{y} v_{0}+\sum_{i=1}^{m} c_{i} \frac{\delta_{y} v_{i}}{v_{i}}\right)
$$

Let $M=K\left(v_{0}, v_{1}, \ldots, v_{m}\right)$ and let

$$
\begin{array}{ll}
u_{0}=-(1 / c) & \text { Trace } v_{0}, \\
u_{i}=\text { Norm } v_{i} & \text { for } i=1, \ldots, m, \\
a_{i}=-c_{i} / c & \text { for } i=1, \ldots, m,
\end{array}
$$

where the Norm and Trace are with respect to $L$ over $K$. We then have

$$
\frac{1}{f}=\delta_{y} u_{0}+\sum_{i=1}^{m} a_{i} \frac{\delta_{y} u_{i}}{u_{i}}
$$

with $u_{0}, \ldots, u_{m}$ in $K$ and $a_{i}$ in $C\left(K,\left\{\delta_{y}\right\}\right)$.

3. Algorithmic considerations. The preceding work was motivated by our desire to develop a decision procedure for finding elementary first integrals. These results show that we need only look for elementary integrals of a prescribed form. In this section we shall discuss the problem of finding an elementary first integral for a two-dimensional autonomous system of differential equations and reduce this problem to that of bounding the degrees of algebraic solutions of this system. 
Let $K=\mathbf{C}(x, y)$, where $x$ and $y$ are transcendental over $\mathbf{C}$, and let $\Delta=\left\{\boldsymbol{\delta}_{x}, \delta_{y}\right\}$, where these derivations are just the usual partial derivatives with respect to $x$ and $y$. Consider the system of differential equations,

$$
\frac{d x}{d t}=P(x, y), \quad \frac{d y}{d t}=Q(x, y),
$$

where $P$ and $Q$ are polynomials in $\mathbf{C}[x, y]$ and let $D=P \delta_{x}+Q \delta_{y}$. We say that (6) has an elementary first integral if there exists an elementary extension $(L, \Delta)$ of $(K, \Delta)$ such that $C(L, \Delta)=C(K, \Delta)$ and $C(L, \Delta) \subsetneq C(L,\{D\})$. The existence of an elementary first integral is intimately related to the existence of an algebraic integrating factor for $Q d x-P d y$. Without explicitly mentioning this 1-form, the following propositions describe this relationship.

Proposition 1. If the equations of (6) have an elementary first integral, then there exists an element $R \neq 0$ algebraic over $K$ such that $D R=-\left(\delta_{x} P+\delta_{y} Q\right) R$.

Proof. Applying the Theorem of $\S 2$, there exist $w_{0}, \ldots, w_{n}$ algebraic over $K$ and $c_{1}, \ldots, c_{n}$ in $C(K, \Delta)$ such that $D w_{0}+\sum_{i=1}^{n} c_{i} D w_{i} / w_{i}=0$ and $\delta w_{0}+\sum_{i=1}^{n} c_{i} \delta w_{i} / w_{i} \neq$ 0 for some $\delta$ in $\Delta$. Let

$$
R_{1}=\delta_{x} w_{0}+\sum_{i=1}^{n} c_{i} \frac{\delta_{x} w_{i}}{w_{i}}, \quad R_{2}=\delta_{y} w_{0}+\sum_{i=1}^{n} c_{i} \frac{\delta_{y} w_{i}}{w_{i}} .
$$

We then have $0=P R_{1}+Q R_{2}$. We may assume that at least one of $P$ or $Q$ is nonzero, say $Q$. Letting $R=R_{1} / Q$, we have $R_{1}=Q R$ and $R_{2}=-P R$. Since $\delta_{x}$ and $\delta_{y}$ commute in any algebraic extension of $K$, we see that $\delta_{y}(Q R)=\delta_{x}(-P R)$. Carrying out this differentiation gives us that $D R=-\left(\delta_{x} P+\delta_{y} Q\right) R$.

Proposition 2. Assume that there exists an element $S \neq 0$, algebraic over $K$ such that $D S=-\left(\delta_{x} P+\delta_{y} Q\right) S$. Then either

(i) there exists $a w$ in $K$ such that $D w=0$ and $\delta w \neq 0$ for $\delta$ in $\Delta$, or

(ii) for any $R \neq 0$ algebraic over $K$ such that $D R=-\left(\delta_{x} P+\delta_{y} Q\right) R$, there exists a $c$ in $\mathbf{C}$ such that $R=c S$ and furthermore $R^{n}$ is in $K$ for some $n$ in $\mathbf{Z}$.

If (i) holds, then the equations in (6) obviously have an elementary first integral. If (i) does not hold, then the equations in (6) have an elementary first integral if and only if there exist $w_{0}, \ldots, w_{n}$ algebraic over $K$ and $c_{1}, \ldots, c_{n}$ in $\mathbf{C}$ such that

$$
\delta_{x} w_{0}+\sum c_{i} \frac{\delta_{x} w_{i}}{w_{i}}=S Q, \quad \delta_{y} w_{0}+\sum c_{i} \frac{\delta_{y} w_{i}}{w_{i}}=-S P
$$

Proof. Let $R \neq 0$ be algebraic over $K$ and satisfy $D R=-\left(\delta_{x} P+\delta_{y} Q\right) R$. Furthermore, assume (i) does not hold, that is, that $D w=0$ implies $w \in \mathbf{C}$ for $w$ in $K$. Since $R / S$ satisfies $D(R / S) /(R / S)=0$ we have $R / S$ is in $\mathbf{C}$, so $R=c S$ for some $c$ in $\mathbf{C}$. Let $E$ be a normal algebraic extension of $K$ containing $R$. For any $K$-automorphism $\sigma$ of $E$, we have $D(\sigma R) / \sigma R=-\left(\delta_{x} P+\delta_{y} Q\right)$. Summing this relation over all $\sigma$ in the galois group of $E$ over $K$, we get

$$
\frac{D(\operatorname{Norm} R)}{\operatorname{Norm} R}=-n\left(\delta_{x} P+\delta_{y} Q\right)
$$


for some $n$ in $\mathbf{Z}$. Therefore $D\left(\right.$ Norm $\left.R / R^{n}\right) /\left(\right.$ Norm $\left.R / R^{n}\right)=0$ so $R^{n}$ is a constant multiple of Norm $R$ and therefore in $K$.

Now, assume that (6) has an elementary first integral. We have shown in the proof of Proposition 1 that there exist $w_{0}, \ldots, w_{n}$ and $R$ algebraic over $K$ and $c_{1}, \ldots, c_{n}$ in $\mathbf{C}$ such that

$$
\delta_{x} w_{0}+\sum_{i=1}^{n} c_{i} \frac{\delta_{x} w_{i}}{w_{i}}=R Q, \quad \delta_{y} w_{0}+\sum_{i=1}^{n} c_{i} \frac{\delta_{y} w_{i}}{w_{i}}=-R P
$$

and

$$
D R=-\left(\delta_{x} P+\delta_{y} Q\right) R .
$$

If (i) does not hold, then by (ii) we have $R=c S$ for some $c$ in $\mathbf{C}$ so

$$
\delta_{x}\left(\frac{w_{0}}{c}\right)+\sum_{i=1}^{n} \frac{c_{i}}{c} \frac{\delta_{x} w_{i}}{w_{i}}=S Q, \quad \delta_{y}\left(\frac{w_{0}}{c}\right)+\sum_{i=1}^{n} \frac{c_{i}}{c} \frac{\delta_{y} w_{i}}{w_{i}}=-S P .
$$

Conversely, if there exist $w_{0}, \ldots, w_{n}$ algebraic over $K$ and $c_{1}, \ldots, c_{n}$ in $\mathbf{C}$ such that

$$
\delta_{x} w_{0}+\sum_{i=1}^{n} c_{i} \frac{\delta_{x} w_{i}}{w_{i}}=S Q \text { and } \delta_{y} w_{0}+\sum_{i=1}^{n} c_{i} \frac{\delta_{y} w_{i}}{w_{i}}=-S P
$$

then

$$
D w_{0}+\sum_{i=1}^{n} c_{i} \frac{D w_{i}}{w_{i}}=P(S Q)-Q(S P)=0
$$

while

$$
\delta w_{0}+\sum_{i=1}^{n} c_{i} \frac{\delta w_{i}}{w_{i}} \neq 0 \text { for some } \delta \text { in } \Delta .
$$

We can then find an elementary extension $(L, \Delta)$ of $(K, \Delta)$ with $C(L, \Delta)=C(K, \Delta)$ such that $L$ contains an element $w$ with $D w=0$ and $\delta w \neq 0$ for some $\delta$ in $\Delta$.

Therefore, to decide if (6) has an elementary first integral, we must:

(A) Decide if $D w=0$ has a nonconstant solution in $\mathbf{C}(x, y)$ and find one if it does.

(B) If $D w=0$ has only constant solutions in $\mathbf{C}(x, y)$, decide if

$$
D S=-\left(\delta_{x} P+\delta_{y} Q\right) S
$$

has a nonconstant algebraic solution $S$ with $S^{n}$ in $\mathbf{C}(x, y)$ for some $n$ in $\mathbf{Z}$ and find one if it does.

(C) If (B) holds, decide if there exist $w_{0}, \ldots, w_{n}$ algebraic over $K$ and $c_{1}, \ldots, c_{n}$ in $\mathbf{C}$ such that

$$
\delta_{x} w_{0}+\sum_{i=1}^{n} c_{i} \frac{\delta_{x} w_{i}}{w_{i}}=S Q \quad \text { and } \quad \delta_{y} w_{0}+\sum_{i=1}^{n} c_{i} \frac{\delta_{y} w_{i}}{w_{i}}=-S P
$$

and find them if they do.

We can solve (C) completely, so we shall deal with it first. Let $k$ be the algebraic closure of $\mathbf{C}(y)$ and let $F=k(x, S)$. Considering $F$ as an ordinary differential field with derivation $\delta_{x}$, we have $C\left(F,\left\{\delta_{x}\right\}\right)=k$. The first step in solving problem (C) is 
to decide if $S Q=\delta_{x} u_{0}+\sum c_{i} \delta_{x} u_{i} / u_{i}$ with $u_{i}$ in $F$ and $c_{i}$ in $k$. Since $F$ is algebraic over $k(x)$, a solution of this latter problem was described in [RISCH: 70]. If no such $u_{i}$ and $c_{i}$ exist, we are done. If $u_{i}$ and $c_{i}$ do exist we can assume that the $c_{i}$ are linearly independent over $\mathbf{Q}$ and that $\delta_{x} u_{i} \neq 0$ for $i=1, \ldots, n$. Next decide if the $c_{i}$ are in $\mathbf{C}$, i.e. $\delta_{y} c_{i}=0$ for all $i$. If some $\delta_{y} c_{i} \neq 0$, then we claim that for any choice of $w_{0}, \ldots, w_{n}$ in $F$ and $d_{1}, \ldots, d_{n}$ in $k$ such that $S Q=\delta_{x} w_{0}+\sum d_{i} \delta_{x} w_{i} / w_{i}$ we have $\delta_{y} d_{i} \neq 0$ for some $i$. To see this assume we have $w_{0}, \ldots, w_{n}$ in $k$ and $d_{i}$ in $\mathbf{C}$ such that $S Q=\delta_{x} w_{0}+\sum d_{i} \delta_{x} w_{i} / w_{i}$ and assume that $c_{1} \notin \mathbf{C}$. We then have

$$
\delta_{x}\left(u_{0}-w_{0}\right)+\sum c_{i} \frac{\delta_{x} u_{i}}{u_{i}}-\sum d_{i} \frac{\delta_{x} w_{i}}{w_{i}}=0 .
$$

If we extend $c_{1}, \ldots, c_{n}$ to a $\mathbf{Q}$-basis of $c_{1} \mathbf{Q}+\cdots+c_{n} \mathbf{Q}+d_{1} \mathbf{Q}+\cdots+d_{m} \mathbf{Q}$ and rewrite the above equation we get

$$
\delta_{x}\left(u_{0}-w_{0}\right)+c_{1} \frac{\delta_{x} u_{1}}{u_{1}}+\sum c_{i} \frac{\delta_{x} v_{i}}{v_{i}}=0,
$$

where the $v_{i}$ are power products of $u_{2}, \ldots, u_{n}, w_{1}, \ldots, w_{m}$. Since all the terms appearing here are algebraic over $k(x)$ and $\delta_{x} x=1$, we have that $u_{i}$ is in $k$ so $\delta_{x} u_{1}=0$, a contradiction. Therefore if some $c_{i}$ is not in $\mathbf{C}$, we can conclude that (6) does not have an elementary integral and we are done. Therefore assume that we have found $u_{0}, \ldots, u_{n}$ algebraic over $F$ and $c_{1}, \ldots, c_{n}$ in $\mathbf{C}$ such that $S Q=\delta_{x} u_{0}+$ $\sum c_{i} \delta_{x} u_{i} / u_{i}$. Consider the expression

$$
I=\delta_{y} u_{0}+\sum c_{i} \frac{\delta_{y} u_{i}}{u_{i}}+S P
$$

Since $\delta_{x}$ and $\delta_{y}$ commute, we have

$$
\delta_{x} I=\delta_{y}\left(\delta_{x} u_{0}+\sum c_{i} \frac{\delta x u_{i}}{u_{i}}-S Q\right)=0 .
$$

Therefore, $I$ is in $k$ and so in some finite extension of $\mathbf{C}(y)$. Now use [RISCH: 70] to decide if there exist $v_{0}, \ldots, v_{m}$ algebraic over $\mathbf{C}(y)$ and $d_{1}, \ldots, d_{m}$ in $\mathbf{C}$ such that

$$
I=\delta_{y} v_{0}+\sum_{i=1}^{m} d_{i} \frac{\delta_{y} v_{i}}{v_{i}}
$$

If such elements exist, we then have

$$
\delta_{x} u_{0}-\delta_{x} v_{0}+\sum c_{i} \frac{\delta_{x} u_{i}}{u_{i}}-\sum d_{i} \frac{\delta_{x} v_{i}}{v_{i}}=\delta_{x} u_{0}+\sum c_{i} \frac{\delta_{x} u_{i}}{u_{i}}=S Q
$$

and

$$
\delta_{y} u_{0}-\delta_{y} v_{0}+\sum c_{i} \frac{\delta_{y} u_{i}}{u_{i}}-\sum d_{i} \frac{\delta_{y} v_{i}}{v_{i}}=I-S P-\left(\delta_{y} v_{0}+\sum d_{i} \frac{\delta_{y} v_{i}}{v_{i}}\right)=-S P
$$

and so we are done. If no such elements exist, then we claim that there are no elements $w_{0}, \ldots, w_{k}$ algebraic over $\mathbf{C}(x, y)$ and $c_{1}, \ldots, c_{k}$ in $\mathbf{C}$ such that

$$
\delta_{y} w_{0}+\sum e_{i} \frac{\delta_{y} w_{i}}{w_{i}}=-S P
$$


If such elements existed, then we would have

$$
\delta_{y} u_{0}+\sum c_{i} \frac{\delta_{y} u_{i}}{u_{i}}+\delta_{y} w_{0}+\sum e_{i} \frac{\delta_{y} w_{i}}{w_{i}}=I .
$$

This implies that $I$ has an antiderivative (with respect to $\delta_{y}$ ) in an elementary extension of $\mathbf{C}(x, y)$. Note that $I$ is algebraic over $\mathbf{C}(y)$ and $\mathbf{C}(x, y)$ is a $\delta_{y}$ elementary extension of $\mathbf{C}(y)$ with new constants. The strong Liouville Theorem of [RISCH: 69] implies that there exist $v_{0}, \ldots, v_{n}$ algebraic over $\mathbf{C}(y)$ and $c_{1}, \ldots, c_{n}$ in $\mathbf{C}$ such that $I=\delta_{y} v_{0}+\sum c_{i} \delta_{y} v_{i} / v_{i}$. This completes the procedure for (C). Note that if $S$ is actually in $\mathbf{C}(x, y)$ then problem (C) always has a positive solution.

We now turn to problems (A) and (B). Let $w$ be an element of $\mathbf{C}(x, y)$ and write $w=\prod_{i=1}^{m} f_{i}^{n}$, with $f_{i}$ irreducible in $\mathbf{C}[x, y]$ and $n_{i}$ in $\mathbf{Z}$. If $D w=0$, we then see $f_{i}$ must divide $D f_{i}$ for each $i$. Let $S$ be an element algebraic over $\mathbf{C}(x, y)$ with $S^{n}$ in $\mathbf{C}(x, y)$ such that $D S=-\left(\delta_{x} P+\delta_{i} Q\right) S$. Write $S=\prod_{i=1}^{m} f_{i}^{r_{i}}$ with $f_{i}$ irreducible in $\mathbf{C}[x, y]$ and $r_{i}$ in $\mathbf{Q}$. Since

$$
\sum_{i=1}^{m} r_{i} \frac{D f_{i}}{f_{i}}=\frac{D S}{S}=-\left(\delta_{x} P+\delta_{y} Q\right)
$$

we again have that each $f_{i}$ divides $D f_{i}$. Results of Darboux [JOU: 79, Theorem 3.3, p. 102 and Lemma 3.53, p. 112] imply that the degree of each $f_{i}$ is bounded. No effective bound is given. This suggests the following problem:

(D) Given $P$ and $Q$ in $\mathbf{C}[x, y]$, let $D=P \delta_{x}+Q \delta_{1}$. Given an effective procedure to find an integer $N$ so that if $f$ is irreducible in $\mathbf{C}[x, y]$ and $f$ divides $D f$, then the degree of $f$ is less than $N$.

Assuming one has a solution for problem (D), one can solve problems (A) and (B). The results of Darboux quoted above imply that if $f_{1}, \ldots, f_{m}$ are irreducible in $\mathrm{C}[x, y]$ and $f_{i}$ divides $D f_{i}$ then either $m<((d+1) d / 2)+2$ where $d=$ $\max (\operatorname{deg} P, \operatorname{deg} Q)$ or there exist integers $n_{i}$, not all zero, such that

$$
n_{1} \frac{D f_{1}}{f_{1}}+\cdots+n_{m} \frac{D f_{m}}{f_{m}}=0 .
$$

In the latter case, we would have $D w=0$ for $w=\prod_{i=1}^{m} f_{i}^{n_{1}}$. To solve problems (A) and (B), note that once we can find $N$ as in (D), the set of coefficients of polynomials $f$ of degree $\leqslant N$ so that $f$ divides $D f$ forms a projective variety which we can construct. We can therefore decide if this variety has fewer than $d(d+1) / 2+2$ points. If it has more points, then we can find them and construct a $w$ in $\mathbf{C}(x, y)$ such that $D w=0$. If it has fewer, then we find all of them, thereby finding all polynomials $f_{i}$ of degree $\leqslant N$ such that $f_{i}$ divides $D f_{i}$. We then decide if we can find $r_{i}$ in $\mathbf{Q}$, not all zero such that

$$
\sum r_{i} \frac{D f_{i}}{f_{i}}=-\left(\delta_{x} P+\delta_{y} Q\right) .
$$

Therefore we have reduced problems (A) and (B) to (D). When $\max (\operatorname{deg} P, \operatorname{deg} Q)$ $=1$, a solution to this problem appears in [JOU: 79, pp. 8-19]. For $\max (\operatorname{deg} P, \operatorname{deg} Q)>1$ no solution seems to be known. Partial results appear in 
[PAIN: 72, vol. I, pp. 173-218, vol. II, pp. 433-458 and POINC: 34, vol. III, pp. 32-97]. Yet even without a solution to problem (D), the above suggests a heuristic method. Arbitrarily fix an integer $N$. Find all irreducible polynomials $f$ of degree $\leqslant N$ such that $f_{i}$ divides $D f_{i}$. If the resulting $D f_{i} / f_{i}$ are linearly dependent over $\mathbf{Z}$, we then can find a $w$ such that $D w=0$. If the $D f_{i} / f_{i}$ are not linearly independent over $\mathbf{Z}$, decide if there exist $r_{i}$ in $\mathbf{Q}$ such that

$$
\sum r_{i} \frac{D f_{i}}{f_{i}}=-\left(\delta_{x} P+\delta_{y} Q\right) .
$$

If such $r_{i}$ exist, let $S=\prod f_{i}^{r_{1}}$ and decide if there exist $w_{0}, \ldots, w_{n}$ algebraic over $\mathbf{C}(x, y)$ and constants $c_{1}, \ldots, c_{n}$ in $\mathbf{C}$ such that

$$
\delta_{x} w_{0}+\sum_{i=1}^{n} c_{i} \frac{\delta_{x} w_{i}}{w_{i}}=S Q, \quad \delta_{y} w_{0}+\sum_{i=1}^{n} c_{i} \frac{\delta_{y} w_{i}}{w_{i}}=-S P .
$$

EXAMPle 1 REVISITED AGAIN. We again consider the system $\dot{x}=a x-b x y$ and $\dot{y}=-c y+d x y$. Letting $D=(a x-b x y) \delta_{x}+(-x y+d x y) \delta_{y}$ we let $N=1$ and look for all polynomials $f=\alpha x+\beta y+\gamma$ of degree $\leqslant 1$ such that $f$ divides $D f$. Since $D f=(\beta d-\alpha b) x y+a \alpha x-c \beta y$, if $f$ divides $D f$ we must have that either $\alpha=0$ or $\beta=0$. In both cases we get $\gamma=0$. Therefore, there are just two first degree polynomials $f, x$ and $y$ such that $f$ divides $D f$. Furthermore,

$$
\frac{D x}{x}=-b y+a \text { and } \frac{D y}{y}=d x-c .
$$

One can check that, unless $b=d=0$, these are linearly independent over $\mathbf{Z}$. We now solve

$$
r_{1} \frac{D x}{x}+r_{2} \frac{D y}{y}=r_{1}(-b y+a)+r_{2}(d x-c)=-(a-b y+(-c+d x))
$$

and find $r_{1}=r_{2}=-1$. Let $S=x^{-1} y^{-1}$ and find $w_{0}, \ldots, w_{n}$ and $c_{1}, \ldots, c_{n}$ constants such that

$$
\begin{aligned}
& \delta_{x} w_{0}+\sum_{i=1}^{n} c_{i} \frac{\delta_{x} w_{i}}{w_{i}}=S Q=-\frac{c}{x}+d, \\
& \delta_{y} w_{0}+\sum_{i=1}^{n} c_{i} \frac{\delta_{y} w_{i}}{w_{i}}=-S P=-\frac{a}{y}+b .
\end{aligned}
$$

We get $w_{0}=d x+b y, w_{1}=x, w_{2}=y, c_{1}=-c, c_{2}=-a$ and so $d x+b y-$ $c \log x-a \log y$ is an elementary first integral.

\section{REFERENCES}

[JOU] J. P. Jouanolou, Equations de Pfaff algébriques, Lecture Notes in Math., vol. 708, Springer-Verlag, Berlin and New York, 1979.

[MACK] C. Mack, Integration of affine forms over elementary functions, Computer Science Department, University of Utah, Technical Report, VCP-39, 1976.

[M.-B.] D. Mordukhai-Boltovski, Researches on the integration in finite terms of differential equations of the first order, Communications de la Société Mathématique de Kharkov, X (1906-1909), pp. 34-64, 231-269. (Russian) Translation of pp. 34-64, B. Korenblum and M. J. Prelle, SIGSAM Bulletin, Vol. 15, No. 2, May 1981, pp. 20-32. 
[MOZI] J. Moses and R. Zippell, An extension of Liouville's Theorem, Symbolic and Algebraic Computation (E. W. Ng, ed.), Springer-Verlag, Berlin and New York, 1979, pp. 426-430.

[PAIN] Oeuvres de Paul Painlevé, Vols. I and II, Editions CNRS, Paris, 1972.

[POINC] Oeuvres de Henri Poincaré, Vol. III, Gauthier-Villars, Paris, 1934.

[PRELLE] M. J. Prelle, Thesis, Rensselaer Polytechnic Institute, 1982.

[RISCH] R. H. Risch, The problem of integration in finite terms, Trans. Amer. Math. Soc. 139 (1969), 167-189.

[RISCH] (1970), 605-608. , The solution of the problem of integration in finite terms, Bull. Amer. Math. Soc. 76

[RITT] J. F. Ritt, On the integrals of elementary functions, Trans. Amer. Math. Soc. 25 (1923), 211-222. [RITT] , Integration in finite terms. Liouville's theory of elementary methods, Columbia Univ. Press, New York, 1948.

[ROS] M. Rosenlicht, On Liouville's theory of elementary functions, Pacific J. Math. 65 (1976), 485-492.

[ROSSIN] M. Rosenlicht and M. Singer, On elementary, generalized elementary, and liouvillian extension fields, Contributions to Algebra, Academic Press, New York, 1977, pp. 329-342.

[SING] M. Singer, Elementary solutions of differential equations, Pacific J. Math. 59 (1975), 535-547.

[SING] __ Functions satisfying elementary relations, Trans. Amer. Math. Soc. 227 (1977), 185-206.

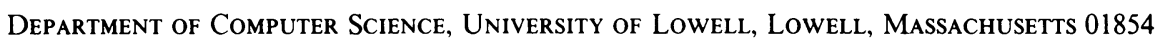

Department of Mathematics, North Carolina State University, Raleigh, North Carolina 27650 